\title{
Active Galactic Nuclei in Dusty Starbursts at z=2: Feedback Still to Kick in
}

Rodighiero, G.; Enia, A.; Delvecchio, I.; Lapi, A.; Magdis, G. E.; Rujopakarn, W.; Mancini, C.; RodriguezMunoz, L.; Carraro, R.; lani, E.

Total number of authors:

21

Published in:

Astrophysical Journal Letters

Link to article, DOI:

$10.3847 / 2041-8213 / a b 222 e$

Publication date:

2019

Document Version

Publisher's PDF, also known as Version of record

Link back to DTU Orbit

Citation (APA):

Rodighiero, G., Enia, A., Delvecchio, I., Lapi, A., Magdis, G. E., Rujopakarn, W., Mancini, C., Rodriguez-Munoz, L., Carraro, R., Iani, E., Negrello, M., Franceschini, A., Renzini, A., Gruppioni, C., Perna, M., Baronchelli, I.,

Puglisi, A., Cassata, P., Daddi, E., ... Silverman, J. (2019). Active Galactic Nuclei in Dusty Starbursts at $z=2$ :

Feedback Still to Kick in. Astrophysical Journal Letters, 877(2), [L38]. https://doi.org/10.3847/2041-8213/ab222e

\section{General rights}

Copyright and moral rights for the publications made accessible in the public portal are retained by the authors and/or other copyright owners and it is a condition of accessing publications that users recognise and abide by the legal requirements associated with these rights.

- Users may download and print one copy of any publication from the public portal for the purpose of private study or research.

- You may not further distribute the material or use it for any profit-making activity or commercial gain

- You may freely distribute the URL identifying the publication in the public portal 


\title{
Active Galactic Nuclei in Dusty Starbursts at $z=2$ : Feedback Still to Kick in
}

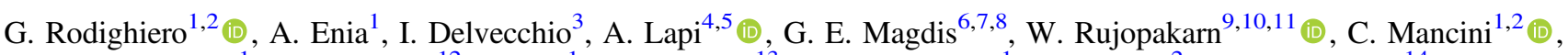 \\ L. Rodríguez-Muñoz $^{1}{ }^{(1 D}$, R. Carraro $^{12}$, E. Iani ${ }^{1}$, M. Negrello ${ }^{13}$, A. Franceschini ${ }^{1}$, A. Renzini ${ }^{2}$ (D), C. Gruppioni ${ }^{14}$, M. Perna ${ }^{15}$,

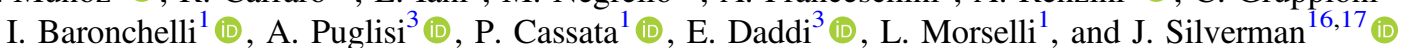 \\ ${ }^{1}$ Dipartimento di Fisica e Astronomia, Universitá di Padova, Vicolo dell'Osservatorio, I-3 35122 Padova, Italy; giulia.rodighiero@unipd.it \\ 2 INAF Osservatorio Astronomico di Padova, vicolo dell'Osservatorio 5, I-35122 Padova, Italy \\ ${ }^{3}$ Laboratoire AIM-Paris-Saclay, CEA/DSM-CNRS-Universit Paris Diderot, Irfu/Service dAstrophysique, CEA-Saclay, Service dAstrophysique, F-91191 Gif-sur- \\ Yvette, France \\ ${ }^{4}$ SISSA, Via Bonomea 265, I-34136 Trieste, Italy \\ ${ }^{5}$ IFPU - Institute for fundamental physics of the Universe, Via Beirut 2, I-34014 Trieste, Italy \\ ${ }^{6}$ Cosmic Dawn Center at the Niels Bohr Institute, University of Copenhagen and DTU-Space, Technical University of Denmark, Denmark \\ ${ }^{7}$ Niels Bohr Institute, University of Copenhagen, Lyngbyvej 2, DK-2100 Copenhagen, Denmark \\ ${ }^{8}$ Institute for Astronomy, Astrophysics, Space Applications and Remote Sensing, National Observatory of Athens, GR-15236 Athens, Greece \\ ${ }^{9}$ Department of Physics, Faculty of Science, Chulalongkorn University, 254 Phayathai Road, Pathumwan, Bangkok 10330, Thailand \\ ${ }^{10}$ National Astronomical Research Institute of Thailand (Public Organization), Don Kaeo, Mae Rim, Chiang Mai 50180, Thailand \\ ${ }^{11}$ Kavli Institute for the Physics and Mathematics of the Universe (WPI),The University of Tokyo Institutes for Advanced Study, The University of Tokyo, Kashiwa, \\ Chiba 277-8583, Japan \\ ${ }^{12}$ Instituto de Física y Astronomía, Universidad de Valparaíso, Gran Bretana 1111, Playa Ancha, Valparaíso, Chile \\ ${ }^{13}$ School of Physics and Astronomy, Cardiff University, The Parade, Cardiff CF24 3AA, UK \\ ${ }^{14}$ Osservatorio di Astrofisica e Scienza dello Spazio Istituto Nazionale di Astrofisica, via Gobetti 93/3, I-40129, Bologna, Italy \\ ${ }^{15}$ INAF - Osservatorio Astrofisico di Arcetri, Largo Enrico Fermi 5, I-50125 Firenze, Italy \\ ${ }^{16}$ Kavli Institute for the Physics and Mathematics of the Universe (WPI), Todai Institutes for Advanced Study, the University of Tokyo, Kashiwanoha, Kashiwa, 277- \\ 8583, Japan \\ ${ }^{17}$ Department of Astronomy, School of Science, The University of Tokyo, 7-3-1 Hongo, Bunkyo, Tokyo 113-0033, Japan \\ Received 2019 March 13; revised 2019 May 13; accepted 2019 May 13; published 2019 June 4
}

\begin{abstract}
We investigate a sample of 152 dusty sources at $1.5<z<2.5$ to understand the connection of enhanced star formation rate (SFR) and black hole accretion rate. The sources are Herschel-selected, having stellar masses $M_{*}>10^{10} M_{\odot}$ and SFR $\left(\sim 100-1000 M_{\odot} \mathrm{yr}^{-1}\right)$ elevated $(>4 \times)$ above the star-forming "main sequence," classifying them as starbursts (SBs). Through a multiwavelength fitting approach (including a dusty torus component), we divided the sample into active SBs (dominated by an active galactic nucleus (AGN) emission, SBs-AGN, $\sim 23 \%$ of the sample) and purely star-forming SBs (SBs-SFR). We visually inspected their Hubble Space Telescope/ultraviolet (UV) rest frame maps: SBs-SFR are generally irregular and composite systems; 50\% of SBs-AGN are instead dominated by regular compact morphologies. We then found archival Atacama Large Millimeter/submillimeter Array continuum counterparts for 33 galaxies (12 SBs-AGN and 21 SBs-SFR). For these sources we computed dust masses, and, with standard assumptions, we also guessed total molecular gas masses. SBs turn into gas-rich systems $\left(f_{\text {gas }}=M_{\text {gas }} /\left(M_{\text {gas }}+M_{*}\right) \simeq 20 \%-70 \%\right)$, and the gas fractions of the two SB classes are very similar $\left(f_{\text {gas }}=43 \% \pm 4 \%\right.$ and $\left.f_{\text {gas }}=42 \% \pm 2 \%\right)$. Our results show that SBs are consistent with a mixture of: (1) highly star-forming merging systems (dominating the SBs-SFR) and (2) primordial galaxies, rapidly growing their $M_{*}$ together with their black hole (mainly the more compact SBs-AGN). Feedback effects have not yet reduced their $f_{\text {gas }}$. Indeed, SBs at $z=2$, with relatively low bolometric AGN luminosities in the range $10^{44}<L_{\mathrm{bol}}(\mathrm{AGN})<10^{46} \mathrm{erg} \mathrm{s}^{-1}$ (compared to bright optical and X-ray quasars), are still relatively far from the epoch when the AGN feedback will quench the SFR in the host and will substantially depress the gas fractions.
\end{abstract}

Key words: galaxies: active - galaxies: evolution - galaxies: formation - galaxies: high-redshift - galaxies: starburst - infrared: galaxies

\section{Introduction}

The existence of a natural correlation between stellar mass and star formation rate (SFR) for the bulk of star-forming galaxies (i.e., the so-called main sequence (MS); Daddi et al. 2007; Magdis et al. 2010; Whitaker et al. 2012; Speagle et al. 2014) and the identification of highly star-forming outliers above it, implies that two main processes of galaxy growth occur: (1) a secular growth along a (quasi-) steady state, and (2) stochastic episodes of major galaxy growth, possibly driven by major mergers that trigger short-lived and intense starbursts (SB). Similarly, two different ways of black hole (BH) growth seem to hold. Likely, secular processes dominate the growth of intermediate-to-low luminosity BHs, e.g., through continuous gas refueling (Mullaney et al. 2012). Instead, the most luminous population of active galactic nuclei (AGNs) may experience a different growth history strictly connected to the SB activity in off-MS galaxies. Indeed, SBs show the following indications of enhanced AGN activity: (1) higher average X-ray luminosities (Rodighiero et al. 2015, and R. Carraro et al., in preparation) (2) higher AGN fraction (up to 50\%$80 \%$ ) both in the local universe (Alexander \& Hickox 2012), and at high-z (Bongiorno et al. 2012; Perna et al. 2018), compared to MS galaxies ( 25\%-30\%, Brusa et al. 2009) This implies that the AGN duty cycle is higher above the MS, and that the black hole accretion rate (BHAR) is more efficient.

Many observational efforts have been devoted to understanding what triggers the fast gas consumption rate in these spectacular sources (Silverman et al. 2015; Cibinel et al. 2018; 
Calabrò et al. 2019). Mergers are often invoked as the more likely mechanism, although it is still not clear if this is sufficient to explain the enhanced star formation of SBs compared to normal galaxies (Silverman et al. 2018). Some authors suggest that the merger mechanism enhancing SFR in many SBs could be responsible also for the AGN ignition (e.g., Di Matteo et al. 2005; Capelo et al. 2015; Steinborn et al. 2018). This scenario is, however, still quite debated (e.g., Mancuso et al. 2016; Wang et al. 2019).

In this Letter, we investigate the properties of SBs in COSMOS field at $1.5<z<2.5$. We study their morphologies, AGN contribution, and molecular gas contents (for a subsample with an Atacama Large Millimeter/submillimeter Array (ALMA) continuum observation) to infer potential observational evidences of feedback induced by AGN.

We adopt a Chabrier (2003) initial mass function assuming a standard cosmology with $H_{0}=70, \Omega_{\lambda}=0.7, \Omega_{0}=0.3$.

\section{Dusty SBs at $1.5<z<2.5$ : Sample Selection}

We adopt the Herschel far-infrared catalog, associated to the COSMOS2015 sample (Laigle et al. 2016), to select a sample of highly star-forming dusty galaxies at the peak of the cosmic SFR density (i.e., $1.5<z<2.5$ ). PACS and SPIRE-Herschel data are originally from the PEP (Lutz et al. 2011) and HerMES (Oliver et al. 2012) surveys, with corresponding photometry extracted with a $24 \mu \mathrm{m}$ priors point-spread function fitting procedure. Sources identification, multiwavelength photometry, $M_{*}$, and redshifts are from the COSMOS2015 sample. SBs are selected to have a SFR well elevated above the MS at $z=2$, at least a factor 4, as in Rodighiero et al. (2011).

\subsection{Spectral Energy Distribution (SED) Fitting: SFR and AGN Computation}

We fit the multiwavelength SEDs of the Herschel sources to derive their physical characterization. We used both the MAGPHYS (da Cunha et al. 2008), and the SED3FIT (Berta et al. 2013) SED-fitting codes, the latter accounting for an additional AGN component (dusty torus). MAGPHYS relies on the energy balance between the dust-absorbed stellar continuum and the reprocessed dust emission at infrared (IR) wavelengths. SED 3FIT combines the emission from stars, dust heated by star formation, and a possible AGN-torus component from the library of Feltre et al. (2012). We fitted each observed SED by using the best available redshift (either spectroscopic or photometric) as input. The SFR was derived from the total IR (rest frame [8-1000] $\mu \mathrm{m}$ ) luminosity taken from the best-fit galaxy SED (subtracted by the AGN luminosity, if present), assuming a Kennicutt (1998) conversion.

Out of 1790 Herschel detected sources at $1.5<z<2.5$, we identified a sample of 164 SBs (see Figure 1). We limit our analysis to $M_{*}>10^{10} M_{\odot}$ (to ensure an unbiased mass complete selection, see Laigle et al. 2016) reducing the SB sample to 152 objects.

\subsection{AGN Classification}

In order to quantify the relative incidence of a possible AGN component, we fitted each individual observed SED. The fit obtained with the AGN is preferred if the reduced $\chi^{2}$ value of the best fit (at $\geqslant 99 \%$ confidence level, on the basis of a Fisher test) is significantly smaller than that obtained from the fit without the AGN (see Delvecchio et al. 2014). From our

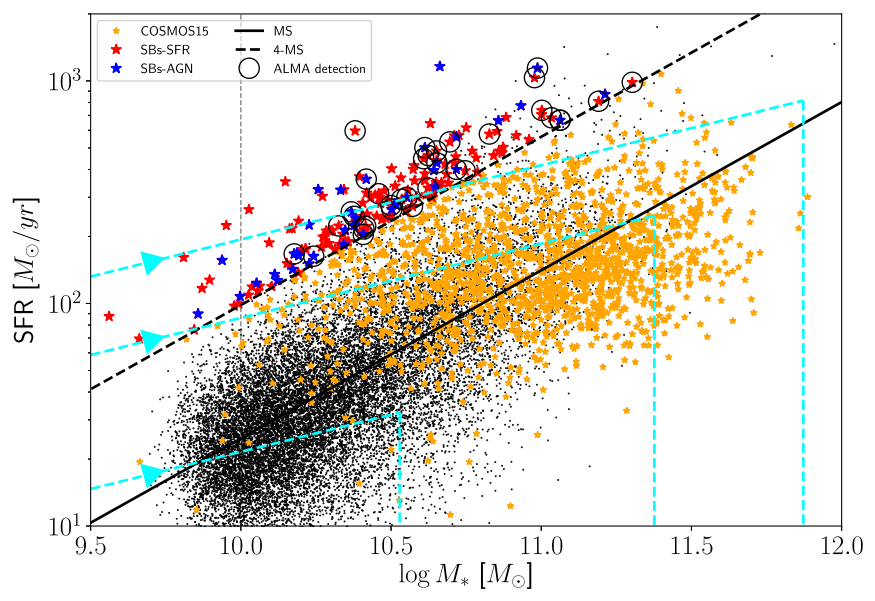

Figure 1. Sample selection in the SFR $-M_{*}$ plane for the COSMOS field at $1.5<z<2.5$. Orange stars mark the original Herschel sample. Blue and red stars show the position of SBs-AGN and SBs-SFR, respectively (see Section 2.1). Black open circles indicate the SB sources with an ALMA detection. Black dots are star-forming BzK (Daddi et al. 2007). We report as dashed cyan lines evolutionary tracks of galaxies (Lapi et al. 2018) starting with different initial SFR and final total $M_{*}$ (time follows the arrows direction, see the discussion in Section 6.2). The vertical black dashed line marks the mass limit of our SBs selection (see the text for details).

analysis, 35 out of 152 SBs (about 23\%) show a significant AGN component. In the following, we will refer to these two classes as SBs with evidences of a nuclear activity (SBs-AGN) and SFR-dominated SBs (SBs-SFR), respectively. We have verified that out of $152 \mathrm{SBs}$ in our starting sample, only eight are classified as X-ray AGN (Laigle et al. 2016), six of which are identified by our AGN classification. This check ensures that we recover the bulk (i.e., $75 \%$ ) of the classical X-ray/AGN selection, ${ }^{18}$ extending the sample to include also the most obscured active sources (e.g., Bongiorno et al. 2012; Gruppioni et al. 2016). Moreover, because at $1.5<z<2.5$ the IRAC bands span the rest frame near-IR where the galaxy stellar emission peaks, to ensure that the AGN classification is not contaminated we performed a further test. Thus, to verify if substantially old stellar populations could bias the AGN selection, we computed the mass-weighted ages of all SBs in our sample with and without the dusty torus component. In spite of a significant scatter ( $\sim 0.3 \mathrm{dex})$, no offset is observed in the measured average stellar ages of the systems, both for SBsSFR and SBs-AGN (probing that the SED3FIT software correctly recovers, in a statistical sense, the near-IR light arising from the stellar populations and from the torus). We have also tested our AGN selection procedure against classical IRAC/Spitzer color-color diagnostics, as the one proposed by Donley et al. (2012), finding that $\sim 80 \%$ of the SBs-AGN fall into the AGN region, while just one of the SBs-SFR are misclassified as AGN (see Figure 2, left panel). A comparison with the average fraction of the luminosities coming from the AGN component at [5-40] $\mu \mathrm{m}$ in the SED modeling, confirms that sources lying above the dashed line in the left panel of Figure 2 have four times larger AGN contributions (25\% versus

\footnotetext{
18 As a consistency check, for X-ray undetected SBs-AGN, we computed the expected [2-10]keV X-ray fluxes, derived from the AGN bolometric luminosities obtained by the SED-fitting procedure described in Section 2.1. By applying a suited bolometric correction (Yang et al. 2018), and a K-correction $=(1+z)^{\Gamma-2}$ (with $\Gamma=1.5$, consistently with the stacked hardness ratios of our sources), we obtain a median $F[2-10] \mathrm{keV}$ on the order of $\sim 1.7 \times 10^{-15} \mathrm{keV}$, right below the limit of the Chandra detectability in this band $\left(\sim 1.9 \times 10^{-15} \mathrm{keV}\right.$; Marchesi et al. 2016).
} 

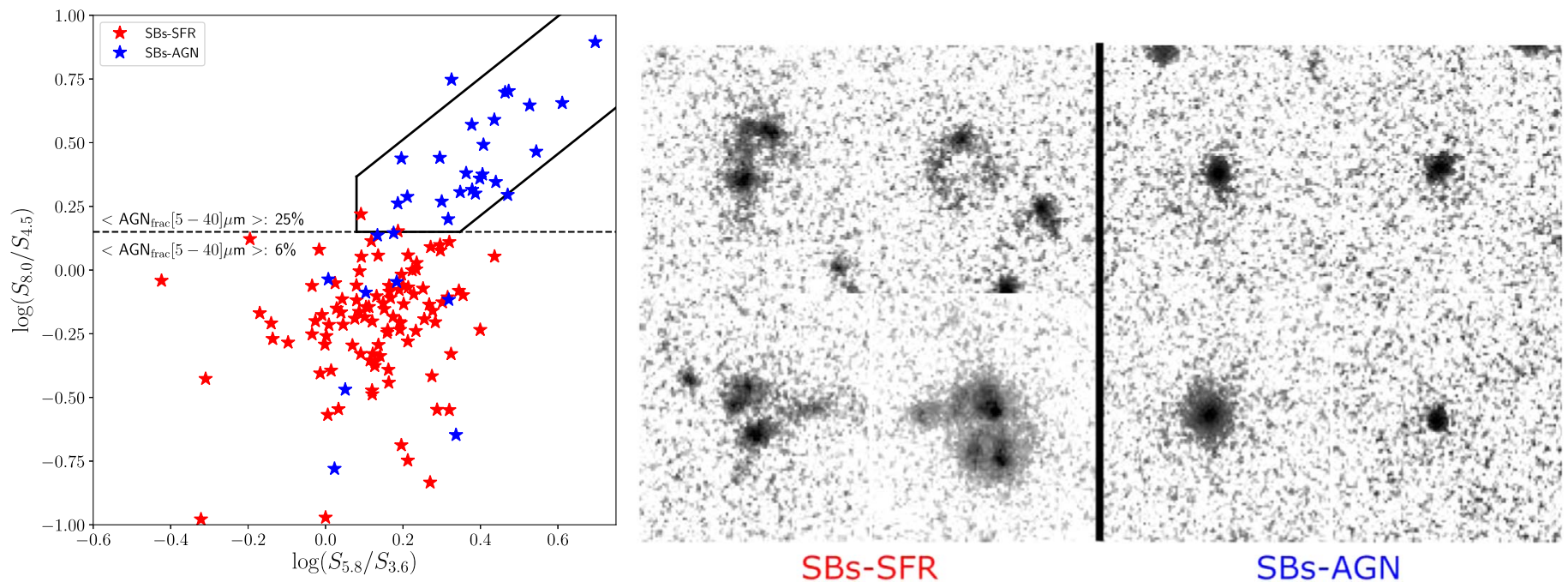

Figure 2. Left panel: color-color IRAC plot of the 152 SBs selected in COSMOS. Blue stars are classified as SBs-AGN (35 in total), red stars as SBs-SFR (117). Black lines mark the position of the AGN selection criteria from Donley et al. (2012). The mean value of the sample AGN contribution (in percentage units) between [5-40] $\mu \mathrm{m}$ over and below this value is reported. Right panel: morphological examples of SFR-dominated starbursts (left) and AGN dominated starbursts (right). HSTACS cutouts have a size of $5^{\prime \prime} \times 5^{\prime \prime}$.

$6 \%$, in terms of the light fraction arising from the torus in the mid-IR) with respect to the sources below the same line. This indicates that the procedure adopted in this work to classify AGN preferentially selects sources dominated by a torus component at mid-IR wavelengths (as expected). The comparison of our AGN selection criterium with the color-color plot presented in Figure 2 (left panel) shows that our SBs-AGN sample is highly reliable ${ }^{19}$ (contamination $\leqslant 20 \%$ ), but not necessarily complete (Donley et al. 2012).

\section{UV-rest Frame Hubble Space Telescope (HST) Morphological Analysis}

We performed a visual inspection on the COSMOS/HST ACS $i$-band images of the 140 (out of 152) SBs for which the data are available (corresponding to the UV-rest frame at $z=2$ ). Excluding undetected sources ( 9 out of 33 SBs-AGN, 39 out of 107 SBs-SFR), this analysis reveals that the two SB classes have statistically different typical morphologies: SBsSFR are disturbed systems (56 out of 68 , i.e., $\sim 82 \% \pm 11 \%$ of the HST detected sample), with evident tidal interaction between multiple components (possibly ongoing mergers or clumpy disks); SBs-AGN are (13 out of 24 detection, $\sim 55 \% \pm 15 \%$ ) dominated by regular compact and symmetric morphologies. We note that for Type 1 sources the HST imaging would be dominated by the AGN outshining the hosts. From our SED fitting, we estimate that just a fraction of the compact SBs-AGN are consistent with a Type 1 classification, lowering to $\sim 34 \%$ the percentage of compact AGN in the sample, which is still significantly higher then the corresponding value among the SBs-SFR $(\sim 18 \%)$. To overcome the uncertainties related to our SED fitting approach on the classification of Type $1 \mathrm{AGN}$, and the shallowness of the X-ray Chandra data in COSMOS, we rely on a stacking procedure to compute separately the average soft $(S)$ and hard $(H) \mathrm{X}$-ray

\footnotetext{
${ }^{19}$ Even if SB templates are a dominant contaminant of mid-IR AGN selection techniques (Rodighiero et al. 2007), Donley et al. (2012) have demonstrated that their conservative selection minimizes the inclusion of purely star-forming LIRGS and ULIRGS, whose templates begin to enter the IRAC selection region only at $z>2.7$.
}

fluxes for the different AGN morphological classes. We used the CSTACK tool ${ }^{20}$ (v3.1, T. Miyaji) and derive the average hardness ratio, $H R=(H-S) /(H+S)$, of our SBs-AGN sample (see details on the procedure in Rodighiero et al. 2015). We found that compact, $i$-band undetected and extended sources have compatible $H R=-0.12 \pm 0.11,0.02 \pm 0.31$, $-0.04 \pm 0.13$, respectively. This result suggests that all AGN in our classification have similar levels of extinction, with column densities on the order of $N_{\mathrm{H}} \sim 10^{22}-10^{23}$ (see Rodighiero et al. 2015). This is inconsistent with a dominant budget of unobscured AGN light arising at UV wavelengths among the compact sources (that could potentially influence rapid morphological transformations in the hosts, e.g., Pierce et al. 2010). Indeed, these $N_{\mathrm{H}}$ values are in the typical range of obscuration observed for Type $2 \mathrm{AGN}$ at $z=2$ (Marchesi et al. 2016).

By also including the $i$-band undetected sources, the fraction of visual compact sources would be $\sim 11 \% \pm 3 \%$ and $\sim 39 \% \pm 7 \%$ for SBs-SFR and SBs-AGN, respectively. Typical examples of the different classes are shown in Figure 2. We caution that this morphological analysis is limited by the shallowness of the COSMOS/HST imaging, but also by the huge dust extinction associated to the SBs, that allows only a minor contribution of un-absorbed UV light to escape the obscured star-forming regions (Puglisi et al. 2017). Despite these important limitations, we interpret our results as a supporting evidence that SBs-AGN are probably more compact and dense sources, likely corresponding to a final merger stage (as many local ULIRGs; Sanders et al. 1988).

\subsection{ALMA Continuum Counterparts}

We searched for possible ALMA millimetric counterparts of the SBs in the public archive, adopting a search radius of 2."5. The final sample includes 33 sources with a clear detection over $3 \sigma$, mostly in band 7 at $870 \mu \mathrm{m}$ (with four undetected sources that we do not include in this work), with few of them detected also in band 3 or 2 . The measurement sets were calibrated

\footnotetext{
${ }^{20}$ http://cstack.ucsd.edu/cstack/
} 
Table 1

Main Observational and Physical parameters of the SBs with an ALMA Continuum Detection (Redshifts between Parentheses are Spectroscopic).

\begin{tabular}{|c|c|c|c|c|c|c|c|c|c|c|c|}
\hline $\begin{array}{l}\text { ID } \\
\text { COSMOS15 }\end{array}$ & $\begin{array}{l}\text { R.A. } \\
\text { (deg) }\end{array}$ & $\begin{array}{l}\text { Decl. } \\
\text { (deg) }\end{array}$ & $z$ & $\begin{array}{c}\log L_{\mathrm{IR}} \\
\left(L_{\odot}\right)\end{array}$ & $\begin{array}{l}\log L_{\mathrm{AGN}} \\
\left(\mathrm{erg} \mathrm{s}^{-1}\right)\end{array}$ & $\begin{array}{c}\log M_{*} \\
\left(M_{\odot}\right)\end{array}$ & $\begin{array}{c}\log M_{\text {gas }} \\
\left(M_{\odot}\right)\end{array}$ & AGN & $\begin{array}{l}F_{870 \mu \mathrm{m}} \\
(\mathrm{mJy})\end{array}$ & $\begin{array}{l}F_{1.3 \mathrm{~mm}} \\
(\mathrm{mJy})\end{array}$ & $\begin{array}{c}F_{3.0 \mathrm{~mm}} \\
(\mathrm{mJy})\end{array}$ \\
\hline 182648 & 150.64316 & 1.558194 & 1.6887 & 11.115 & 45.595 & 10.4616 & 9.840 & yes & $0.49 \pm 0.10$ & $\ldots$ & $\ldots$ \\
\hline 221280 & 149.76807 & 1.617000 & 2.3220 & 11.185 & 43.945 & 10.4703 & 10.282 & yes & $1.11 \pm 0.22$ & $\cdots$ & $\cdots$ \\
\hline 280968 & 149.79010 & 1.711870 & 1.7844 & 11.343 & $\ldots$ & 10.4035 & 10.384 & no & $1.33 \pm 0.25$ & $\cdots$ & $\cdots$ \\
\hline 323041 & 149.81653 & 1.779770 & 2.0933 & 11.244 & 44.135 & 10.5122 & 10.018 & yes & $1.38 \pm 0.46$ & $\cdots$ & $\cdots$ \\
\hline 349784 & 150.48938 & 1.821710 & 1.9693 & 11.100 & $\cdots$ & 10.8425 & 10.628 & no & $1.59 \pm 0.34$ & $\cdots$ & $\cdots$ \\
\hline 506667 & 150.72984 & 2.071170 & 2.4433 & 11.200 & $\ldots$ & 10.5955 & 10.590 & no & $2.23 \pm 0.77$ & $\ldots$ & $\cdots$ \\
\hline 524710 & 149.76853 & 2.099614 & 2.1059 & 11.321 & $\cdots$ & 10.4187 & 10.214 & no & $1.72 \pm 0.23$ & $\cdots$ & $\cdots$ \\
\hline 571598 & 150.61642 & 2.167971 & 1.5052 & 11.167 & $\cdots$ & 10.8600 & 10.792 & no & $5.39 \pm 0.23$ & $\cdots$ & $\cdots$ \\
\hline 578926 & 150.40103 & 2.180390 & $(2.3341)$ & 11.567 & $\ldots$ & 10.9570 & 10.617 & no & $2.05 \pm 0.55$ & $\ldots$ & $\ldots$ \\
\hline 600601 & 150.13265 & 2.211946 & 1.9875 & 10.752 & 45.605 & 11.1349 & 11.081 & yes & $8.27 \pm 0.44$ & $2.46 \pm 0.10$ & $\cdots$ \\
\hline 605409 & 149.76813 & 2.219876 & (1.7766) & 11.112 & $\cdots$ & 10.8708 & 10.585 & no & $3.78 \pm 1.03$ & $\ldots$ & $\cdots$ \\
\hline 745498 & 150.46551 & 2.429549 & 1.6332 & 10.731 & $\cdots$ & 10.7144 & 10.355 & no & $3.47 \pm 0.34$ & $\ldots$ & $\cdots$ \\
\hline 747590 & 150.22447 & 2.433010 & 1.6351 & 10.903 & $\cdots$ & 10.6513 & 10.285 & no & $1.60 \pm 0.24$ & $\cdots$ & $\cdots$ \\
\hline 752016 & 150.33683 & 2.439920 & 2.2682 & 11.585 & $\cdots$ & 10.7232 & 10.665 & no & $4.50 \pm 0.31$ & $\cdots$ & $\ldots$ \\
\hline 754372 & 150.06907 & 2.444010 & 2.4355 & 11.439 & 45.595 & 11.0108 & 10.468 & yes & $4.86 \pm 0.65$ & $\cdots$ & $0.275 \pm 0.065$ \\
\hline 769248 & 150.25528 & 2.466839 & $(2.2640)$ & 10.903 & $\cdots$ & 10.5134 & 10.629 & no & $3.80 \pm 0.42$ & $\cdots$ & $\ldots$ \\
\hline 794848 & 150.09341 & 2.507339 & 2.1990 & 11.365 & 45.975 & 10.7362 & 10.648 & yes & $3.09 \pm 0.25$ & $\cdots$ & $\ldots$ \\
\hline 810228 & 150.11307 & 2.528020 & 2.0167 & 11.264 & $\cdots$ & 10.6265 & 10.687 & no & $\ldots$ & $\cdots$ & $0.137 \pm 0.060$ \\
\hline 815012 & 150.60329 & 2.536536 & $(2.2872)$ & 11.119 & $\cdots$ & 11.1034 & 10.692 & no & $6.70 \pm 0.35$ & $\cdots$ & $\ldots$ \\
\hline 818426 & 150.72202 & 2.541904 & 2.2664 & 11.607 & 45.935 & 10.6405 & 10.807 & yes & $1.01 \pm 0.30$ & $\ldots$ & $\ldots$ \\
\hline 842595 & 149.99796 & 2.578227 & 2.4200 & 11.693 & 44.205 & 10.7809 & 10.732 & yes & $1.97 \pm 0.32$ & $\cdots$ & $\ldots$ \\
\hline 902320 & 150.03726 & 2.669600 & (1.5990) & 10.274 & $\ldots$ & 10.9826 & 11.162 & no & $6.41 \pm 0.39$ & $\ldots$ & $0.147 \pm 0.057$ \\
\hline 917423 & 149.99218 & 2.693436 & 2.1284 & 11.330 & $\ldots$ & 10.7933 & 10.581 & no & $1.77 \pm 0.34$ & $\ldots$ & $\ldots$ \\
\hline 917546 & 150.16165 & 2.691588 & (1.9745) & 10.937 & 44.785 & 10.4437 & 10.156 & yes & $1.39 \pm 0.25$ & $\cdots$ & $\cdots$ \\
\hline
\end{tabular}

running the data reduction scripts with the Common Astronomy Software Applications (CASA). Fluxes and associated errors were evaluated with CASA, by fitting the emissions with a bidimensional Gaussian, and are reported in Table 1. For the 33 ALMA detected sources, we included the sub-mm fluxes in the observed SED and we performed a second fitting run, as described in Section 2.1. The updated physical parameters are reported in Table 1.

\section{Results}

In this Section, we report the main results of our analysis of the gas masses computed for the SBs with an ALMA continuum detection. Out of 33 sources, 12 objects turn into SBs-AGN, thus representing $36 \%$ of our sample. We note that this limited ALMA detected data set is quite representative of the whole SB population in this redshift range (in terms of $M_{*}$, elevation above the MS and AGN content; see Figure 1).

\subsection{Comparison Sample}

For comparative analyses, we use the reference sample compiled by Sargent et al. (2014) and Perna et al. (2018), including "typical" star-forming galaxies at $z \leqslant 3$ with measurements of their CO luminosity. Local ULIRGs and high- $z$ SBs with a CO detection are added. Detailed references for the various samples included are reported in Sargent et al.
(2014) and Perna et al. (2018). We added the recent compilation of SBs by Silverman et al. (2015) and Silverman et al. (2018), at $z \sim 1.6$.

\subsection{Dust and Molecular Gas Masses}

Dust masses ( $\left.M_{\text {dust }}\right)$ have been derived following the procedure described in Magdis et al. $(2012,2017)$ by fitting the SED in the IRAC-ALMA observed frame with the Draine \& Li (2007) models. The total gas mass ( $M_{\text {gas }}$, which incorporates both the molecular and atomic phases) can be inferred from the dust mass through the metallicity dependent gas-to-dust ratio (GDR; e.g., Eales et al. 2010; Magdis et al. 2012): $M_{\text {gas }}=M_{\text {dust }} / \operatorname{GDR}(Z)$.

For consistency with $M_{\text {gas }}$ estimates of SBs in the literature, we adopt GDR $\approx 30$, that based on Magdis et al. (2012) corresponds to a CO-to- $M_{\text {gas }} \quad \alpha_{\text {co }}$ conversion factor of $0.8 M_{\odot} /\left(\mathrm{K} \mathrm{km} \mathrm{s}^{-1} \mathrm{pc}^{2}\right)$, typically used for strong $\mathrm{SBs}$. For completeness we also infer $M_{\text {gas }}$ estimates with GDR $\approx 95$ that corresponds to solar gas-phase metallicity that could be considered as a lower limit (on metallicity, hence the upper limits on GDR and thus on $M_{\text {gas }}$ ) for dust-obscured SBs (e.g., Puglisi et al. 2017). These $M_{\text {gas }}$ estimates are consistent (within $0.15 \mathrm{dex}$ ) with the average $M_{\mathrm{gas}}$ estimates derived based on the monochromatic flux densities in the R-J tail of the SED (one or more of 870,1300 , and $3000 \mu \mathrm{m}$ in our case) and the recipe presented in Scoville et al. (2017). 

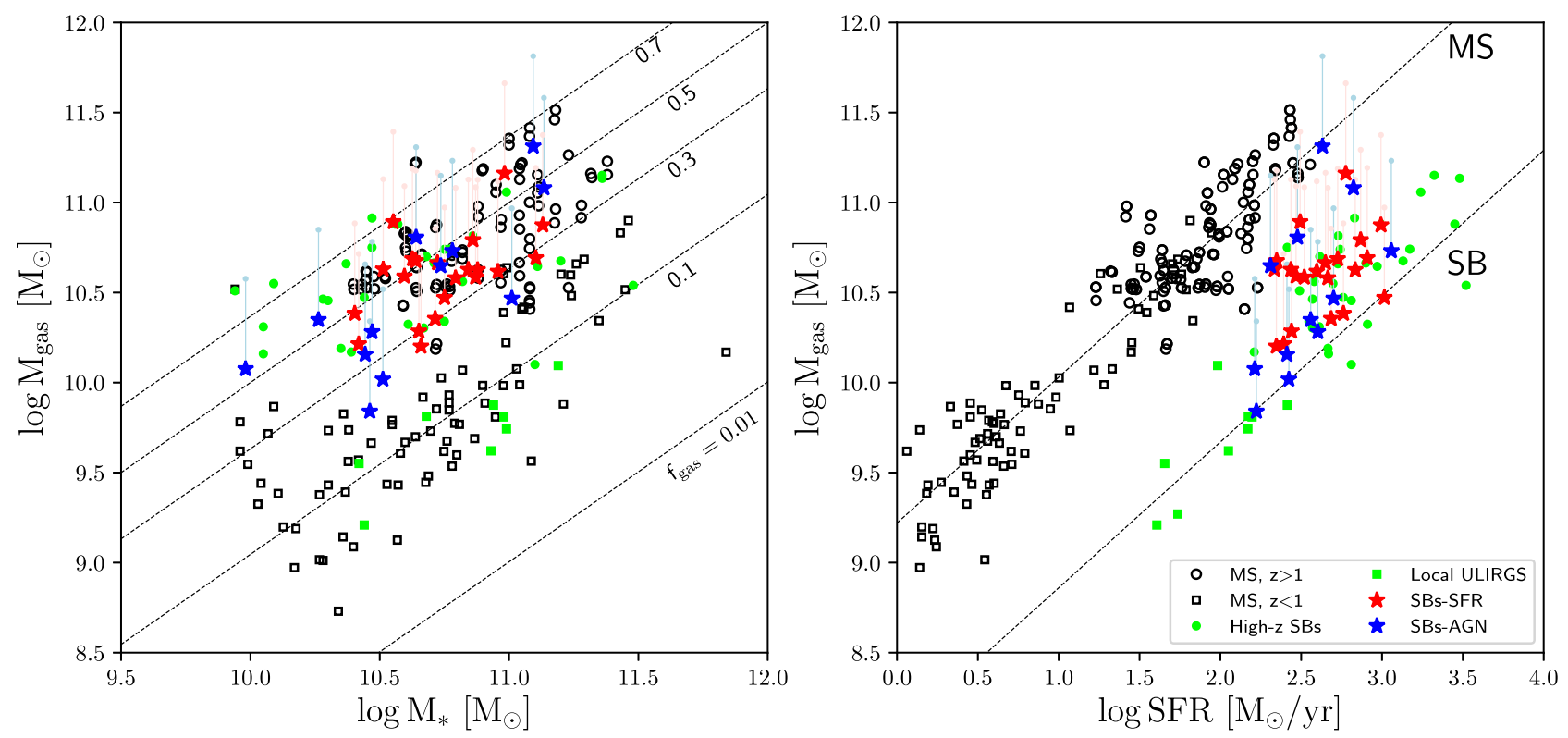

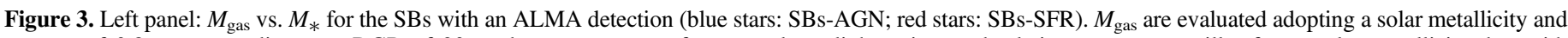

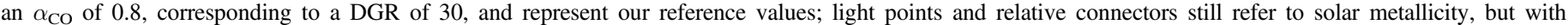

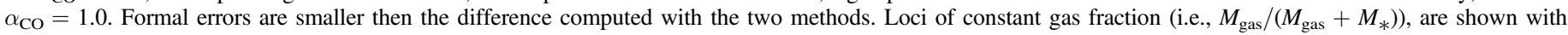

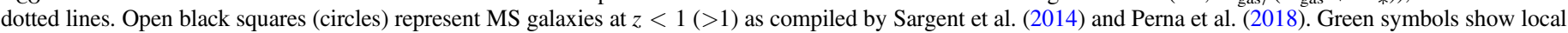

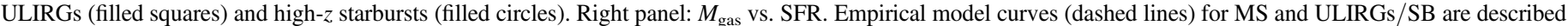
in Sargent et al. (2014).

\subsection{Gas Masses and Gas Fractions in SBs-AGN and SBs-SFR}

We report in Figure 3 (left panel) the gas masses of the SBs as a function of their $M_{*}$, divided into SBs-AGN and SBs-SFR (blue and red filled stars, respectively). The SB population is dominated by gas-rich galaxies, with gas fractions (defined as $\left.f_{\text {gas }}=M_{\text {gas }} /\left(M_{\text {gas }}+M_{*}\right)\right)$ spanning the range $20 \%-70 \%$. This is similar to the typical $f_{\text {gas }}(\sim 50 \%)$ of the normal star-forming sources at similar $z$ (open circles). Local star-forming galaxies (both MS and ULIRGs/SBs) are instead much gas poorer, with $f_{\text {gas }} \sim 10 \%$, as expected on the basis of the observed gas fraction evolution with cosmic time (e.g., Magdis et al. 2012; Tacconi et al. 2018, and references therein).

When looking at the separate gas fractions of SBs-AGN and SBs-SFR, we do not observe a significant difference, providing average values of $f_{\text {gas }}=43 \% \pm 4 \%$ and $f_{\text {gas }}=42 \% \pm 2 \%$ for the two classes, respectively.

To shed more light on our analysis in the most efficient starforming sources, we study (right panel of Figure 3) the distribution of $M_{\text {gas }}$ as a function of SFR, in order to compare the star formation efficiency (i.e., $\mathrm{SFE}=\mathrm{SFR} / M_{\mathrm{gas}}$ ) of SBsAGN and SBs-SFR. Our sample turns to be "SFR-selected" by construction, with SFR $\geqslant 150 M_{\odot} \mathrm{yr}^{-1}$ due to the requirement of being Herschel selected (see Figure 1). We then observe that SBs lie on a contiguous sequence of increasing SFE that fills the gap between the two paradigmatic sequences of normal galaxies and ULIRG/SBs (dotted lines, as from Sargent et al. 2014), usually interpreted as the main loci of two extremely different SF modes. Our results support recent works suggesting the existence of a continuous increase in SFE with elevation from the MS, as opposed to a bimodal distribution (Silverman et al. 2018).

\section{Discussion}

\subsection{Comparison with the Merger Triggered SB-Quasar (QSO) Evolutionary Sequence}

As mentioned in Section 1, we can compare our results with the expectations of the AGN-galaxy co-evolutionary scenario, that predicts a luminous IR phase of buried SMBH growth, coexisting with a SB (likely arising from a merger; Donley et al. 2018; Koss et al. 2018) before feedback phenomena deplete the cold molecular gas reservoir of the galaxy and an optically luminous QSO shines out (Hopkins et al. 2008). On one side, we have qualitatively observed that SBs including an AGN have on average more compact and nucleated UV-rest frame morphologies with respect to "inactive" SBs, suggesting that they are kept in a different dynamical evolutionary phase. This could correspond to the key transition when the late mergers trigger a high SFR, before the fully developed AGN phase. Simulations and observations, indeed, suggests a temporal delay between the peak of the SFR and the peak of the BHAR (Rodighiero et al. 2015; Bergvall et al. 2016; Lapi et al. 2016). On the other side, we did not find a significant reduction of gas fractions in the SBs-AGN hosts compared to "inactive" SBsSFR. We argue that, if major mergers are the main triggering mechanism of obscured BHAR in SBs, feedback phenomena (producing large outflows from the central $\mathrm{BH}$ ) are not efficient in removing significant amount of molecular gas in the host galaxies.

\subsection{SBs as Primordial Galaxies}

An alternative interpretation for the properties of the off-MS galaxies is provided by an in situ co-evolution scenario (Lapi et al. 2018), envisaging high-z galaxy evolution to be mainly ruled by the interplay between in situ processes like gas infall, compaction, star formation, accretion onto the hosted central SMBHs, and related feedback processes (with wet galaxy 
mergers having a minor role at $z \geqslant 1$ ). In this framework $z \sim 2$ sources with SFR $\geqslant$ a few $10^{2} M_{\odot}$ constitute the progenitor of local massive spheroids with stellar mass $M_{*} \geqslant 10^{11} M_{\odot}$. During their star-forming phase, lasting some $10^{8} \mathrm{yr}$, these galaxies feature a nearly constant SFR and a linearly increasing stellar mass. In the SFR- $M_{*}$ diagram they follow an almost horizontal track (see the cyan tracks in Figure 1) while moving toward the galaxy MS locus; there they will have acquired most of their mass before being quenched by energy/momentum feedback from the SMBH.

Being in the early stages of their evolution, the SBs can host only a rather small SMBH, originating a bolometric AGN luminosity $L_{\mathrm{AGN}}$ that is weaker than that $L_{\mathrm{SFR}}$ associated to the $\mathrm{SFR}$ in the host. However, the $\mathrm{BH}$ mass is expected to grow exponentially generating a noticeable statistical variance in $L_{\mathrm{AGN}}$ at given SFR. As a consequence, in the SFR versus $L_{\mathrm{AGN}}$ plane, $\mathrm{SBs}$ are expected to populate a strip parallel to the $L_{\mathrm{AGN}}$ axis, and located to the left of the locus where $L_{\mathrm{SFR}}=L_{\mathrm{AGN}}$ (see Figure 4 and Bianchini et al. 2018). The hosted AGNs are expected to be obscured and have a luminosity that is still not powerful enough to originate substantial feedback effects on the interstellar medium (ISM) of the host galaxy; thus, the SFR and the gas mass of the host are still not much affected. The compact morphologies of SBs-AGN (possibly linked to a forming bulge) could indicate that these sources are observed when the host stellar mass and the BH mass are reaching their maximum, just before the feedback gets into action and the BHAR and SFR gets quenched (Lapi et al. 2014); further size evolution of the stellar component may be induced by the feedback itself and by late-time mass addition from dry mergers (see Lapi et al. 2018).

\section{Summary}

In conclusion, the results presented in this work are consistent with the idea that the SB population could be filled by a mixture of (1) a class of highly star-forming merging sources (preferentially among the SBs-SFR) and (2) primordial galaxies, quickly accreting their $M_{*}$ together with their $\mathrm{BH}$ (mainly the SBs-AGN). If the level of AGN luminosity (proportional to BHAR) is also correlated with the power of feedback, then IR-selected SBs are mainly low-luminosity AGN, and feedback effects have not yet started to eventually reduce the gas fraction of the objects (as observed for local Seyfert in a similar AGN luminosity range; Rosario et al. 2018). As time passes, the galaxies will get older, the luminosity of the AGN will overwhelm that of the SFR in the host (moving toward the bottom-right region of Figure 4), removing gas and dust from the ISM and quenching the SFR and the accretion itself. The systems will then shine as a bright optical quasar (blue symbols in Figure 4), before turning into passive sources.

To better constrain the dominant nature of SB (mergers versus in situ formation and evolution), much larger samples are required to provide a statistical description of the gas content in this population and to understand the impact of the co-evolving obscured central activity. An important discriminating factor will be provided by future spatially resolved kinematic studies of the stellar or gas components (James Webb Space Telescope, ALMA), currently available for just few sources (e.g., Silverman et al. 2018).

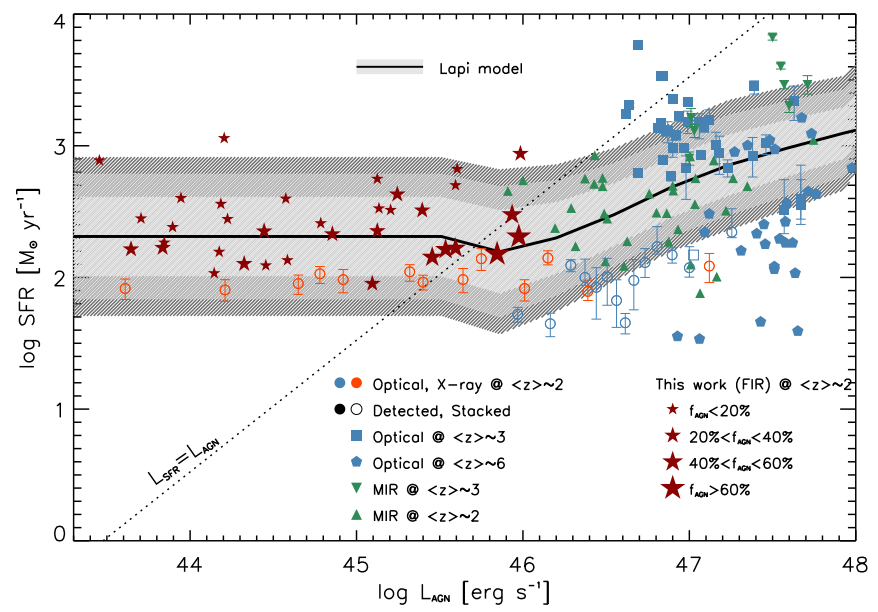

Figure 4. Co-evolution plane between bolometric AGN luminosity and SFR in the hosts $z>1.5$. The gray shaded areas show the average locus of the evolutionary tracks from the model of Lapi et al. (2018), adopting an SFR detection threshold around $10^{2} M_{\odot} \mathrm{yr}^{-1}$. Orange symbols refer to X-ray AGNs, green to mid-IR selected AGNs, and blue to optically selected QSOs. Complete references are reported in Bianchini et al. (2018). We include all SBs-AGN from our original sample (red filled stars): size of the symbol is proportional to the AGN luminosity contribution in the $5-40 \mu \mathrm{m}$ range, derived from $S E D 3 F I T$. SFR for the SBs in our sample is derived from $L_{\mathrm{IR}}$ in Table 1.

G.R., C.M., A.R., and A.P. acknowledge support from PRIN-SKA ESKAPE-HI (PI: L. Hunt) and from the STARS@UniPD grant. G.R. and A.L. are supported by PRIN MIUR 2017 prot. 20173ML3WW_002 Opening the ALMA window on the cosmic evolution of gas, stars and supermassive black holes. M.P. acknowledges support from the ESO Scientific Visitor Programme. G.E.M. acknowledges support from the Villum Fonden research grant 13160, the Cosmic Dawn Center of Excellence, the ERC Consolidator grant funding scheme (project ConTExt, grant No. 648179). We thank Marcella Brusa for fruitful discussions This Letter makes use of the following ALMA data: ADS/JAO.ALMA\#2011.0.01234.S, 2013.1.00034.S, 2015.1.00137.S, 2015.1.00207.S, 2015.1.00568.S, 2016.1.00171.S,2016.1.00478.S, 2016.1.00646.S, and 2016.1.00804.S. ALMA is a partnership of ESO (representing its member states), NSF (USA) and NINS (Japan), together with NRC (Canada), MOST and ASIAA (Taiwan), and KASI (Republic of Korea), in cooperation with the Republic of Chile. The Joint ALMA Observatory is operated by ESO, AUI/NRAO, and NAOJ.

\section{ORCID iDs}

G. Rodighiero (1) https://orcid.org/0000-0002-9415-2296

A. Lapi (1) https://orcid.org/0000-0002-4882-1735

W. Rujopakarn (1) https://orcid.org/0000-0002-0303-499X

C. Mancini (1) https://orcid.org/0000-0002-4297-0561

L. Rodríguez-Muñoz 1 https://orcid.org/0000-00020192-5131

A. Renzini (i) https://orcid.org/0000-0002-7093-7355

I. Baronchelli (1) https://orcid.org/0000-0003-0556-2929

A. Puglisi (i) https://orcid.org/0000-0001-9369-1805

P. Cassata (ㄱ) https://orcid.org/0000-0002-6716-4400

E. Daddi i https://orcid.org/0000-0002-3331-9590

J. Silverman (1) https://orcid.org/0000-0002-0000-6977

\section{References}

Alexander, D. M., \& Hickox, R. C. 2012, NewAR, 56, 93 
Bergvall, N., Marquart, T., Way, M. J., et al. 2016, A\&A, 587, A72 Berta, S., Lutz, D., Santini, P., et al. 2013, A\&A, 551, A100 Bianchini, F., Fabbian, G., Lapi, A., et al. 2018, arXiv:1806.06516 Bongiorno, A., Merloni, A., Brusa, M., et al. 2012, MNRAS, 427, 3103

Brusa, M., Fiore, F., Santini, P., et al. 2009, A\&A, 507, 1277

Calabrò, A., Daddi, E., Puglisi, A., et al. 2019, A\&A, 623, A64

Capelo, P. R., Volonteri, M., Dotti, M., et al. 2015, MNRAS, 447, 2123 Chabrier, G. 2003, PASP, 115, 763

Cibinel, A., Daddi, E., Sargent, M. T., et al. 2018, arXiv:1809.00715 da Cunha, E., Charlot, S., \& Elbaz, D. 2008, MNRAS, 388, 1595 Daddi, E., Dickinson, M., Morrison, G., et al. 2007, ApJ, 670, 156 Delvecchio, I., Gruppioni, C., Pozzi, F., et al. 2014, MNRAS, 439, 2736 Di Matteo, T., Springel, V., \& Hernquist, L. 2005, Natur, 433, 604

Donley, J. L., Kartaltepe, J., Kocevski, D., et al. 2018, ApJ, 853, 63

Donley, J. L., Koekemoer, A. M., Brusa, M., et al. 2012, ApJ, 748, 142

Draine, B. T., \& Li, A. 2007, ApJ, 657, 810

Eales, S. A., Smith, M. W. L., Wilson, C. D., et al. 2010, A\&A, 518, L62 Feltre, A., Hatziminaoglou, E., Fritz, J., \& Franceschini, A. 2012, MNRAS, 426,120

Gruppioni, C., Berta, S., Spinoglio, L., et al. 2016, MNRAS, 458, 4297

Hopkins, P. F., Hernquist, L., Cox, T. J., \& Kereš, D. 2008, ApJS, 175, 356

Kennicutt, R. C., Jr. 1998, ARA\&A, 36, 189

Koss, M. J., Blecha, L., Bernhard, P., et al. 2018, Natur, 563, 214

Laigle, C., McCracken, H. J., Ilbert, O., et al. 2016, ApJS, 224, 24

Lapi, A., Aversa, R., \& Danese, L. 2016, in Proc. Interplay between Local and Global Processes in Galaxies, ed. S. F. Sanchez, C. Morisset, \& G. Delgado-Inglada

Lapi, A., Pantoni, L., Zanisi, L., et al. 2018, ApJ, 857, 22

Lapi, A., Raimundo, S., Aversa, R., et al. 2014, ApJ, 782, 69

Lutz, D., Poglitsch, A., Altieri, B., et al. 2011, A\&A, 532, A90
Magdis, G. E., Daddi, E., Béthermin, M., et al. 2012, ApJ, 760, 6 Magdis, G. E., Daddi, E., Sargent, M., et al. 2012, ApJL, 758, L9

Magdis, G. E., Rigopoulou, D., Daddi, E., et al. 2017, A\&A, 603, A93

Magdis, G. E., Rigopoulou, D., Huang, J.-S., et al. 2010, MNRAS, 401, 1521

Mancuso, C., Lapi, A., Shi, J., et al. 2016, ApJ, 833, 152

Marchesi, S., Lanzuisi, G., Civano, F., et al. 2016, ApJ, 830, 100

Mullaney, J. R., Daddi, E., \& Béthermin, M. 2012, ApJL, 753, 30

Oliver, S. J., Bock, J., Altieri, B., et al. 2012, MNRAS, 424, 1614

Perna, M., Sargent, M. T., Brusa, M., et al. 2018, arXiv:1807.03378

Pierce, C. M., Lotz, J. M., Primack, J. R., et al. 2010, MNRAS, 405, 718

Puglisi, A., Daddi, E., Renzini, A., et al. 2017, ApJL, 838, L18

Rodighiero, G., Brusa, M., Daddi, E., et al. 2015, ApJL, 800, L10

Rodighiero, G., Cimatti, A., Franceschini, A., et al. 2007, A\&A, 470, 21

Rodighiero, G., Daddi, E., Baronchelli, I., et al. 2011, ApJL, 739, L40

Rosario, D. J., Burtscher, L., Davies, R. I., et al. 2018, MNRAS, 473, 5658

Sanders, D. B., Soifer, B. T., Elias, J. H., et al. 1988, ApJ, 325, 74

Sargent, M. T., Daddi, E., Béthermin, M., et al. 2014, ApJ, 793, 19

Scoville, N., Lee, N., Vanden Bout, P., et al. 2017, ApJ, 837, 150

Silverman, J. D., Daddi, E., Rodighiero, G., et al. 2015, ApJL, 812, L23

Silverman, J. D., Rujopakarn, W., Daddi, E., et al. 2018, ApJ, 867, 92

Speagle, J. S., Steinhardt, C. L., Capak, P. L., \& Silverman, J. D. 2014, ApJS, 214,15

Steinborn, L. K., Hirschmann, M., Dolag, K., et al. 2018, arXiv:1805.06902

Tacconi, L. J., Genzel, R., Saintonge, A., et al. 2018, ApJ, 853, 179

Wang, L., De Lucia, G., Fontanot, F., \& Hirschmann, M. 2019, MNRAS, 482,4454

Whitaker, K. E., van Dokkum, P. G., Brammer, G., \& Franx, M. 2012, ApJL, 754, L29

Yang, G., Brandt, W. N., Vito, F., et al. 2018, MNRAS, 475, 1887 